\title{
Intégration à la société québécoise par l'apprentissage de la langue
}

\author{
par Marie-Françoise Fayolle
}

Salvador, Iran, Nicaragua, Bulgarie, Vietnam, Chili, Guatemala, Laos... nous pourrions continuer cette liste de pays où règnent des conflits ouverts ou latents, où la guerre, la souffrance, la faim et souvent la violation des droits de l'Homme obligent les individus de ces pays à fuir leur propre terre pour gagner un pays d'accueil où ils pourront espérer trouver la paix, la liberté et une certaine sécurité économique.

Ces hommes, femmes et enfants arrivent au Québec seuls ou avec une partie de leur famille et une des premières barrières qu'ils devront affronter est bien sûr, celle de la langue. Avant d'aller plus loin, il est important de noter qu'il y a une différence entre les personnes immigrantes ayant un statut de résident permanent avec celles arrivant directement aux frontières en revendiquant le statut de réfugié. Pour les premières, le gouvernement a mis en place, depuis 1968, les COFI (Centre d'Orientation et de Formation des Immigrants) qui permettent aux personnes immigrantes de suivre une formation en français, à temps plein, durant 30 semaines. Pour les secondes, l'accessibilité aux cours de langues est plus difficile. A cause de leur statut de revendicateurs, ils n'ont pas droit d'aller dans les COFI à temps plein. Différents cours de formation ont donc été mis en place pour faciliter l'apprentissage de la langue aux revendicateurs. Toutefois, malgré l'augmentation de groupes où les cours de jour et de soir vont être offerts, les ressources sont souvent insuffisantes pour permettre de répondre adéquatement aux multiples besoins d'une clientèle adulte.

Le Comité d'Education aux adultes (CEDA) est un de ces organismes qui offre depuis plusieurs années des cours de français langue seconde aux personnes nouvellement arrivées au Québec. Oeuvrant dans un quartier populaire où

\section{... les ressources sont souvent insuffisantes} pour permettre de répondre

$$
\begin{gathered}
\text { adéquatement aux } \\
\text { multiples besoins } \\
\text { d'une clientèle } \\
\text { adulte. }
\end{gathered}
$$

la majorité de la population connaît des difficultés économiques importantes (une grande partie de notre populationcible est prestataire de l'assurancechômage ou du bien-être-social), nous avons été confrontés au fait que de nombreuses communautés culturelles s'installaient dans le quartier (asiatique, antillaise, latino-américaine...) ainsi que dans les quartiers environnants.

Même si tous ces gens vivent une situation identique de déracinement, d'adaptation à une nouvelle culture et de confrontation des différences sociales et culturelles, il reste que chaque personne apporte avec elle un bagage de vie unique qui diffère de celui des autres.

Les groupes sont constitués, pour les cours de débutants, de 20 personnes (norme fixée par le Ministère des Communautés Culturelles et de l'Immigration qui nous subventionne). L'âge des participants varie de 18 à 65 ans. On retrouve dans nos groupes un plus grand nombre de femmes. Un service de halte-garderie gratuit pour les personnes ayant de jeunes enfants facilite l'accès aux cours de français, plus particulièrement aux femmes étant donné que ce sont très souvent elles qui ont la responsabilité des enfants dans les communautés que nous accueillons. Par ailleurs, le fait de suivre ces cours et d'avoir accès à une halte-garderie leur permet de briser l'isolement dans lequel elles sont souvent confinées.

Un aspect qui aura un impact important sur l'apprentissage est, celui lié à la scolarité. Dans un même groupe, nous avons des adultes très scolarisés (niveau universitaire ou collégial) et des personnes analphabètes dans leur propre langue. Défi de taille lorsqu'on sait qu' un grand nombre de personnes analphabètes auront peur d'exprimer cet état par crainte d'être refusées dans le groupe. Les enseignantes devront 
donc trouver des moyens permettant à l'ensemble du groupe d'apprendre le français sans se sentir dévalorisé ou à l'inverse survalorisé.

La pédagogie employée dans les cours devra donc prendre en compte cette réalité tout en respectant le rythme d'apprentissage de chaque personne. Les outils utilisés devront être très visuels. On favorisera l'acquisition de la langue à travers des situations de vie courantes qui rendront les participants plus autonomes et ce, le plus vite possible.

Toutefois, lorsque nous travaillons avec des personnes mois scolarisées, il faut avant même de penser à apprendre la langue, travailler au niveau de la mise en confiance. Ces personnes, qu'elles soient jeunes ou âgées, ont malheureusement la certitude qu'elles ne réussiront pas à apprendre le français: honte de parler, honte devant les autres plus scolarisés, elles auront tendance à se replier et à s'isoler au sein même de la classe ou d'abandonner leur formation. Il faut donc beaucoup de patience, de temps, de valorisation pour redonner à ces personnes la confiance qu'elles ont perdue. Il faut aussi s'assurer que le groupe générera de lui-même de l'entraide, du soutien et de la solidarité. L'enseignante ne peut à elle seule redonner confiance à un individu. C'est aussi dans un contexte plus global que ce dernier apprendra à s'ouvrir et à faire tomber ses propres blocages. Certains participants qui suivaient un cours de 12 heures par semaine n'ont réussi à dire leur premier mot qu'un ou deux mois après le début des cours. C'est dire la lenteur parfois des progrès et l'ampleur des blocages que l'on peut retrouver chez une personne.

Les contraintes extérieures accentuent considérablement les difficultés d'apprentissage de l'ensemble des participants: statut précaire par rapport à leur demande d'immigration, difficultés d'intégration et d'adaptation, coupure avec le pays de provenance et bien souvent séparation avec la famille, adaptation au climat, différences culturelles importantes... etc. Ces situations créent beaucoup d'insécurité, de peur et de malaise. Enfin, des difficultés seront

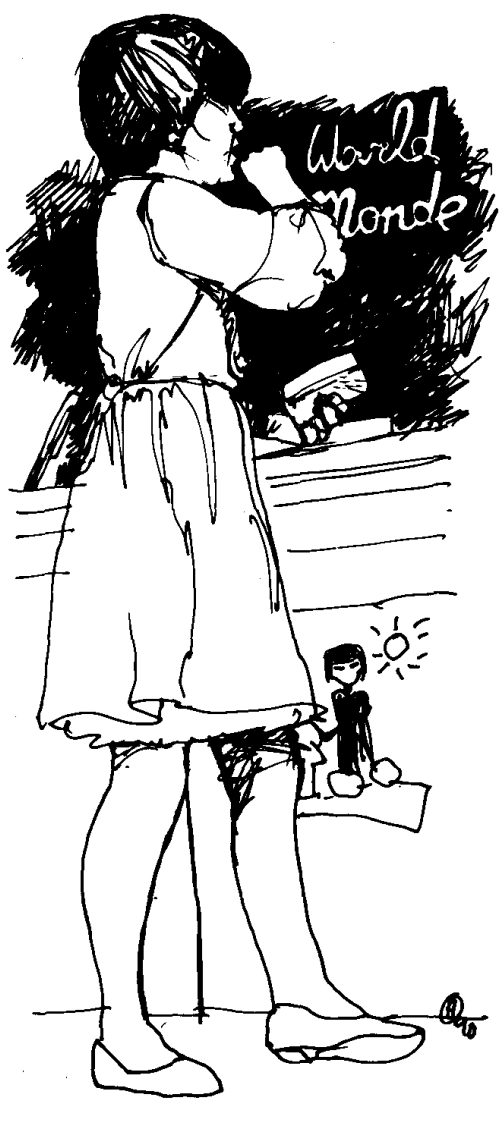

rencontrées de part la nécessité pour la personne immigrante de travailler et d'étudier en même temps. Lorsqu'on passe huit heures dans une manufacture ou une usine, il devient difficile voire impossible de se concentrer, le soir venu sur un banc de classe, et ce, malgré l'utilisation de diverses méthodes. La fatigue, le stress auront raison de la meilleure volonté, l'échec sera souvent vécu comme personnel alors qu'il faudrait plutôt mettre en cause le contexte d'apprentissage (réalité économique, conditions de logement, adaptation... etc.).

L'apprentissage du français ne doit pas être le seul objectif poursuivi. L'intégration des nouveaux arrivants à la société d'accueil doit être tout autant travaillée. En effet, dans la plupart des cas, les participants se retrouveront dans un quartier où il $\mathrm{y}$ a une grande concentration d'immigrants de leur propre communauté. Cela reste un réflexe normal lorsqu'on arrive dans un nouveau pays de se regrouper avec les individus dont la culture, la langue, les traditions sont les mêmes. D'autre part, il faut dire que bien souvent la population de souche aura quelques réticences à accepter les personnes immigrantes parce que cellesci semblent trop différentes et que tout ce qui est étranger fait parfois peur. Parce que notre centre est un groupe d'éducation aux adultes, nous pouvons favoriser l'intégration à travers différentes activités où québécois d'origine et allophones pourront se rencontrer, se parler et mieux se - connaître.

Le centre offre des cours d'alphabétisation pour francophones, des ateliers d'alimentation-santé, d'exercices en douceur, d'artisanat, de céramique, de menuiserie... etc. Le nombre de participants francophones est donc assez important. Par l'intermédiaire de sorties, les groupes seront invités à participer à des activités communes telles que. des sorties à la cabane à sucre, des visites dans le quar- 
tier ou à l'extérieur de Montréal, des danses marquant les événements annuels (Halloween, Noël, fête du printemps...) des repas multi-culturels et enfin, un camp d'hiver qui permet aux personnes de vivre 3 jours (à prix modique) dans un camp de plein air afin de pratiquer des jeux et activités de saison. L'expérience, à ce jour, est assez concluante car les rapports tissés entre les participants francophones et allophones ont permis une meilleure connaissance mutuelle. Pour les personnes francophones nées ici, ces gens venus d'ailleurs ont maintenant une histoire, souvent difficile, ils ne sont plus perçus comme des "voleurs de job" mais plutôt comme des individus à la recherche d'une terre d'asile et de paix. C'est à partir de ces activités que l'on remarquera que les préjugés entretenus des deux côtés vont peu à peu tomber et

\section{Pour les personnes francophones nées ici,}

ces gens venus d'ailleurs ont maintenant une histoire, souvent difficile, ils ne sont plus perçus comme des "voleurs de job" mais plutôt comme des individus à la recherche
d'une terre
d'asile et de paix. faire place à des liens plus centrés vers la découverte de l'autre dans sa globalité.

Malgré ces efforts d'intégration et de sensibilisation qui ont lieu dans plusieurs groupes et à plusieurs niveaux de notre société, il est difficile pour une personne immigrante de comprendre clairement la spécificité du Québec. Les immigrants, majoritairement concentrés à Montréal sont régulièrement confrontés à l'existence des deux langues: anglais et français. Très souvent, ils vont trouver des emplois dans des manufactures où la langue parlée sera uniquement l'anglais. Ces gens qui font l'effort d'apprendre le français sont alors confrontés à l'utilisation d'une autre langue que celle qu'ils apprennent. En dehors du milieu du travail, lorsqu'ils posent des questions d'information dans la rue, beaucoup de nos participants qui ne connaissent pas du tout l'anglais se font régulièrement répondre en anglais par des québécois francophones et, parce qu'ils ont un accent, on a tendance à croire que leur langue est l'anglais. Comment ne pas être perdu dans un tel contexte? Il est évident que le seul moyen pour que tous ces gens possèdent réellement la langue française est de la vivre (autant au travail, à l'école que dans le quartier...) mais comment le faire lorsque dans bien des cas, la langue du travail est l'anglais, la langue du quartier est l'anglais et que bien des francophones ne font pas l'effort (par manque de sensibilisation) de leur parler en français.

Beaucoup d'immigrants ont déjà été en contact avec le français d'outre-mer par des méthodes audios. Cela peut entraîner une certaine confusion dans leur esprit, entre le français de France et le français du Québec. Le décalage est grand car les différences dans les accents, expressions et structures de phrases sont énormes. Une différence existe aussi entre le français qu'on apprend à l'école et celui que l'on entend dans la rue... Comment arriver à leur faire comprendre tous ces différents accents, ces régionalismes, ces expressions qui font la richesse d'une langue? Il faut toujours préciser dans les cours que la langue du Québec n'est pas moins bonne que la langue de France, que les différences sont réelles mais qu'il ne
L'apprentissage du français ne doit pas être le seul objectif poursuivi. L'intégration des nouveaux arrivants à la société d'accueil doit être tout autant travaillée.

faut pas dénigrer la langue française du Québec.

Changer de pays implique pour tous ces gens, peu importe leur pays d'origine, de faire face à un grand nombre d'obstacles. La route est longue avant de pouvoir parler d'une effective intégration au milieu social et économique du pays d'accueil et ce n'est parfois qu'à travers la deuxième génération qu'elle se fera.

Pourtant la première génération ne devrait pas être sacrifiée. Nous avons tout à gagner en tant que membres d'une même société que la population immigrante s'intègre le mieux possible à la société d'accueil et ceci sans perdre leur propre spécificité (culture, traditions, valeurs...). Cela implique que des politiques claires d'intégration soient mises de l'avant mais surtout qu'elles soient portées par l'ensemble des milieux de notre société (travail, syndicat, école, etc.), que des campagnes de sensibilisation, des programmes de formation adéquats (sans distinction de statut) soient aussi mis en place afin de permettre à tous ces nouveaux québécois de construire avec nous une société plus juste.

Les personnes immigrantes ne demandent pas mieux que de s'intégrer à notre pays, nous nous devons de relever ce défi afin d'espérer qu'un jour les mots tels que préjugés et racisme disparaissent de notre langage.

Marie-Françoise Fayolle enseigne le français langue seconde au CEDA. 